\title{
The efficacy and safety of Xuesaitong injection combined with western medicines in the treatment of ischemic stroke: an updated systematic review and meta-analysis
}

\author{
Ling Feng ${ }^{1}$, Xiao-Jun $\mathrm{Wu}^{2}$, Tian $\mathrm{Cao}^{3}, \mathrm{Bo} \mathrm{Wu}^{3}$ \\ ${ }^{1}$ West China School of Nursing, Sichuan University/Department of Neurology, West China Hospital, Sichuan University, Chengdu, China; \\ ${ }^{2}$ Kunming Pharmaceutical, Inc., Kunming, China; ${ }^{3}$ Department of Neurology, West China Hospital, Sichuan University, Chengdu, China \\ Contributions: (I) Conception and design: XJ Wu, T Cao, B Wu; (II) Administrative support: T Cao, B Wu; (III) Provision of study materials or \\ patients: T Cao, B Wu; (IV) Collection and assembly of data: L Feng, XJ Wu; (V) Data analysis and interpretation: T Cao; (VI) Manuscript writing: \\ All authors; (VII) Final approval of manuscript: All authors. \\ Correspondence to: Tian Cao; Bo Wu. Department of Neurology, West China Hospital, Sichuan University, 37 Guo Xue Xiang, Chengdu 610041, \\ China. Email: caotiansweety@163.com; dr.bowu@hotmail.com.
}

Backgroundk Due to constantly emerging new studies on Xuesaitong injection (XST) agent, it is necessary to summarize and analyze the latest published clinical research data to re-evaluate the clinical efficacy and safety of XST. This study mainly aimed to evaluate the clinical efficacy and safety of XST combined with western medicines (WM) for ischemic stroke treatment.

Methods: The related databases were searched to collect all randomized controlled trials (RCTs) comparing the combination of XST and WM with WM alone for the treatment of patients with ischemic stroke. Participant, intervention, comparison, outcome and study type (PICOS) was the inclusion criteria of all studies. The search for and data extraction of the included studies was performed by 2 researchers, who then conducted quality evaluation. The Cochrane Collaboration's risk of bias tool was used for the article evaluation. The software RevMan 5.3 and Stata 15.0 were used for pooled analysis.

Results: A total of 12 RCTs were included involving 1,769 patients with ischemic stroke, which combined XST with WM therapy. Moreover, the funnel plot showed that there was no obvious publication bias in the included studies. Meta-analysis showed that the total effective rate in the XST combined with WM group was higher than that in the $\mathrm{WM}$ alone group [odds ratio $(\mathrm{OR})=4.04,95 \%$ confidence interval (CI): 2.86 to $5.73, \mathrm{P}<0.001]$. The National Institutes of Health Stroke Scale (NIHSS) score in the XST combined with WM group was significantly lower than that in the WM alone group [weighted mean difference (WMD) $=-3.17,95 \%$ CI: -4.14 to $-2.20, \mathrm{P}<0.001]$. In addition, XST combined with WM significantly reduced the plasma viscosity, fibrinogen level, whole blood high shear viscosity, and whole blood low shear viscosity in patients with ischemic stroke $(\mathrm{P}<0.01)$. For the comparison of adverse reactions, the results showed that the adverse reactions among patients with ischemic stroke in the XST combined with WM group and in the WM group did not differ significantly.

Discussion: Compared with single WM medication, XST combined with WM improves the total clinical effective rate, promotes the recovery of patients' neurological function, and has few adverse reactions and high safety in clinical application.

Keywords: Xuesaitong injection (XST); ischemic stroke; systematic review; meta-analysis

Submitted Jun 23, 2021. Accepted for publication Aug 05, 2021.

doi: $10.21037 /$ apm-21-1828

View this article at: https://dx.doi.org/10.21037/apm-21-1828 


\section{Introduction}

Ischemic stroke, also called cerebral vascular accident (CVA), is an acute cerebrovascular disease characterized by cerebral ischemia and hypoxia, leading to focal neurological deficits (1). Epidemiological surveys show that ischemic stroke is mostly found in middle-aged and elderly people and its high incidence rate $(24.6 \%)$, disability rate $(62 \%)$, and mortality rate $(11.4 \%)$ seriously affect people's lives. Currently, the main drugs for treating ischemic stroke are anticoagulative agents, early thrombolysis, and other western medicines (WM). Nevertheless, most people treated with WM still retain at substantial risk of clinically important stroke events due to insufficient inhibition of platelets (2). With the advancement of traditional Chinese medicine (TCM), therapeutic effects of TCM, especially that of promoting blood circulation and removing blood stasis, provide a new approach for the treatment of ischemic stroke.

According to TCM, ischemic stroke belongs to the category of "stroke", and the predominant symptom is blood stasis. As an important therapeutic strategy of TCM, the promotion of blood circulation and removal blood stasis therapy is widely applied in the treatment of acute ischemic stroke $(3,4)$, which can dredge veins, improve the state of blood concentration, adhesion, aggregation, and coagulation, increase the supply of blood and oxygen to the brain, and also rapidly inhibit the progression of ischemic stroke (5-7). Overall, promoting blood circulation and removing blood stasis can reduce the recurrence rate of thrombus, has a good prognosis, and effectively avoids the shortcomings of WM treatment.

Xuesaitong injection agent (XST) includes XST, Sanqi Panax Notoginseng for injection. Its curative effect mainly comes from Panax notoginseng saponins (PNS), including PNS R1, ginsenoside Rg1, Rb1, RD, and RE (8). The clinical use of intermediates of PNS began in the 1970s in terms of "Xuesaitong". Previous research data (9) have shown that PNS can promote the repair of nervous system function, protect cranial nerves, significantly increase the level of superoxide dismutase (SOD), scavenge superoxide anion, reduce oxygen consumption of brain cells, and prolong the cell lifespan (10). It is summarized that PNS has significant therapeutic effects and multiple pharmacological actions including anti-platelet, anti-inflammation, antiischemia, anti-arrhythmia, anti-hyperplasia, anticoagulant, antithrombotic, antiatherosclerosis, lipid-lowering, vasodilative and promoting angiogenesis effects. It is noted that ANTXR cell adhesion molecule 2 (ANTXR2), four and a half LIM domain 3 (FHL3), prolylcarboxypeptidase (PRCP), transmembrane immune signaling adaptor Tyrobp (TYROBP), TATA-box binding protein associated factor 9b (TAF9B), fibroblast growth factor receptor 2 (FGFR2), BAF chromatin remodeling complex subunit (BCL11B), RB1-inducible coiled-coil 1 (RB1CC1) and muscleblindlike splicing regulator 2 (MBNL2) are the pivotal genes and possible action sites of XST in treating ischemic cerebrovascular. As one of the TCMs, XST can improve hemorheology (reducing fibrinogen level and whole blood viscosity) and prevent platelet aggregation to protect vascular endothelial cells and inhibit thrombosis (11). Because of its remarkable therapeutic effect and few adverse reactions, XST has been widely used in the treatment of acute ischemic stroke. At present, there have been 3 metaanalyses published in 2015 or 2019 showing that XST is an effective treatment for ischemic stroke (12-14). However, the clinical efficacy and safety of XST combined with western medicines (WM) for ischemic stroke treatment remains unclear. Therefore, it is necessary to summarize and analyze the latest published clinical research data and collect high-quality research results to re-evaluate the clinical efficacy and safety of XST combined with western medicines (WM), to provide evidence-based information for clinical treatment of ischemic stroke.

We present the following article in accordance with the PRISMA reporting checklist (available at https://dx.doi. org/10.21037/apm-21-1828).

\section{Methods}

\section{Literature search}

The databases of China National Knowledge Infrastructure (CNKI), Wanfang, VIP, PubMed, Embase, and Web of Science were searched by 2 researchers to collect all randomized controlled trials (RCTs) of XST for treating ischemic stroke from 1 January 2015 to 6 January 2020. The search terms included: Xuesaitong, panax notoginsenosides, brain infarction, cerebral ischemic stroke, cerebral infarction, ischemic cerebrovascular disease, ischemic cerebral diseases, infarct of brain, and cerebral ischemia.

\section{Inclusion criteria}

The following criteria were adopted: (I) participants: patients diagnosed with ischemic stroke; (II) study 
type: RCTs; (III) interventions and comparisons: RCTs comparing the combination Xuesaitong and WM to WM alone, such as aspirin, troxerutin injection, glucose injection, venoruton injection, atorvastatin calcium, etc.; (IV) outcomes: total effective rate, National Institutes of Health Stroke Scale (NIHSS) score, fibrinogen levels, plasma viscosity, whole blood high shear viscosity, whole blood low shear viscosity, adverse reactions, and so on; (V) author's affiliations: second-class and upwards hospitals; (VI) sample size $\geq 45$; (VII) time publication: 2015-2020.

\section{Exclusion criteria}

Studies were excluded if any of the following criteria were met: (I) absence of control group; (II) original materials had not been published; (III) unclear analyzed outcomes; (IV) not RCTs; (V) unclear or incomplete related data, and so on; (VI) control group was TCM.

\section{Selection of included studies}

Titles and abstracts were independently read by 2 researchers to screen out irrelevant articles and full texts were read to evaluate whether the articles fit the inclusion criteria. Any differences of opinion were discussed or resolved by a third researcher.

\section{Assessment of risk of bias}

The Cochrane Collaboration's risk of bias tool (Copenhagen: The Nordic Cochrane Center, The Cochrane Collaboration) was used for the article evaluation. The evaluation items included 7 parts: sequence generation (selection bias); allocation concealment (selection bias); blinding of participants and personnel (performance bias); blinding of outcome assessment (detection bias); incomplete outcome data (attrition bias); selective outcome reporting (reporting bias); and other sources of bias (other bias). Each aspect had 3 levels: "low risk", "unclear risk", and "high risk". In addition, the efficacy and safety outcomes were also assessed.

\section{Statistical analysis}

The software Stata 15.0 (StataCorp., College Station, TX, USA) was used to analyze the data. Continuous variables were measured by weighted mean difference (WMD) through the average value and standard deviation (SD), dichotomous outcomes were measured by the odd ratio (OR), and both were calculated with $95 \%$ confidence interval (95\% CI). Heterogeneity analysis was assessed by calculating $\chi^{2}$ and $\mathrm{I}^{2}$. When the test level was $\alpha=0.1$, that is $\mathrm{P} \leq 0.1$ or $\mathrm{I}^{2} \geq 50 \%$, it indicated heterogeneity among the studies, and the random-effects model was selected. When $\mathrm{P}>0.1$ or $\mathrm{I}^{2}<50 \%$, it indicated little heterogeneity, and the fixed-effects model was selected. Subgroup analysis was carried out according to possible heterogeneity factors, sensitivity analysis was used to analyze the influence of each literature on the meta-analysis results, and the funnel plot, Begg's and Egger's tests were used to evaluate the publication bias, where $\mathrm{P}<0.05$ was considered evidence of study effects in all analyses.

\section{Results}

\section{Search results}

A total of 1,829 articles were retrieved via searching the CNKI, Wanfang, VIP, Embase, PubMed, and Web of Science databases. After reading the titles and abstracts, the following articles were excluded: repetitive articles $(\mathrm{n}=831), \mathrm{XST}$ as a control $(\mathrm{n}=371)$, reviews and comments $(n=29)$, pharmaceutical economics research $(n=32)$, nonRCTs ( $\mathrm{n}=218$ ), and studies from hospitals below secondclass $(n=139)$. Totally, 209 articles were initially identified for inclusion in the study. After reading full texts, 129 articles failed to explicitly mention random allocation, 32 articles had a sample size of less than 45, 31 articles had incomplete data information, 5 studies (15-19) compared XST alone with WM, and finally, 12 articles were included. The screening process is shown in Figure 1. The 12 RCTs (20-31) included a total of 1,769 patients, with 889 patients in the XST combined WM treatment group and 885 patients in the WM alone group. These 12 studies compared XST combined WM with WM alone. The basic characteristics of the included studies are summarized in Table 1. All included studies reported specific random methods. The quality evaluation results of the included studies are presented in Figure 2.

\section{Total effective rate}

A total of 11 articles (20-22,24-31) reported the total effective rate of XST combined with WM in the treatment of stroke, and pooled analysis was performed as a binary variable. Heterogeneity test analysis showed that there 


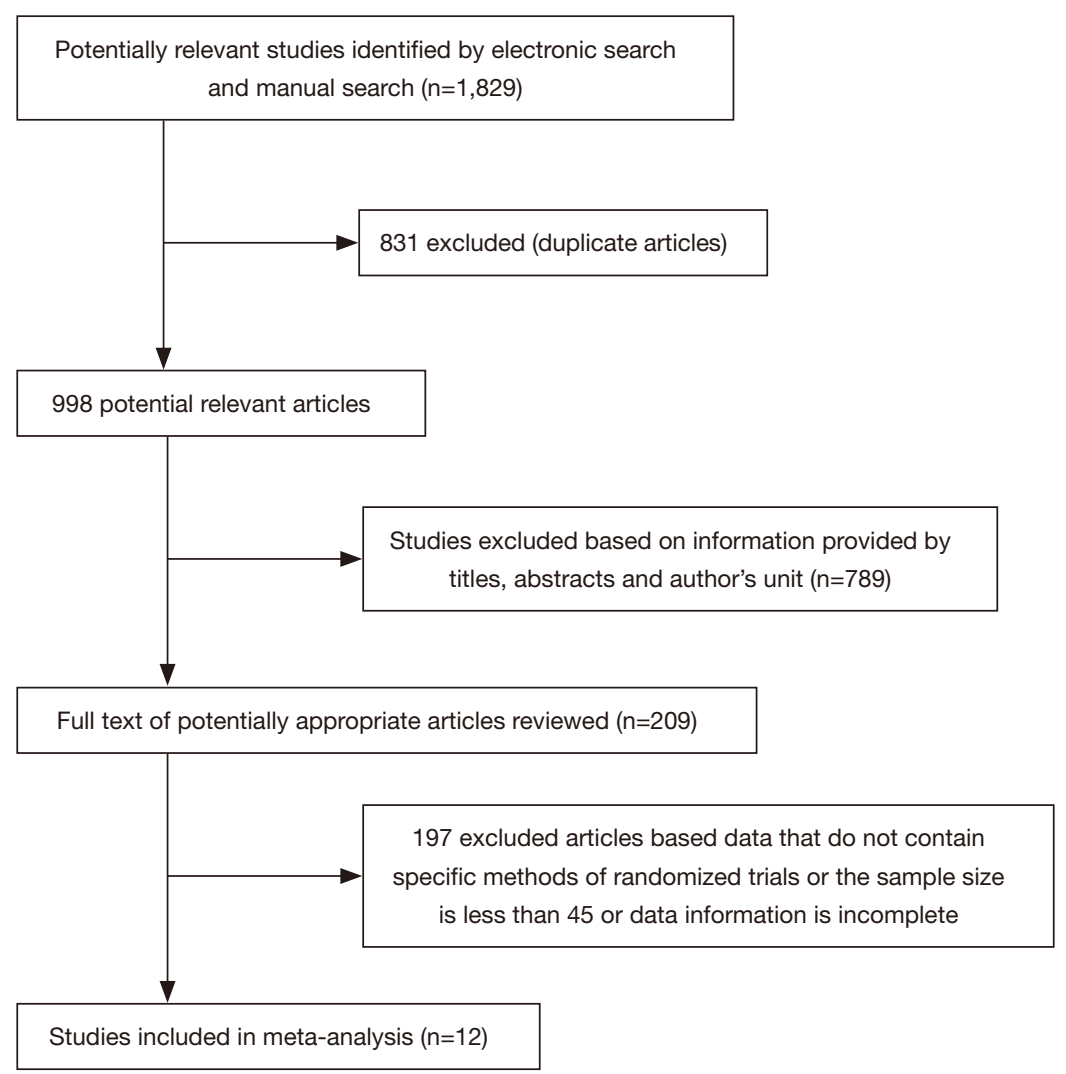

Figure 1 Flow chart of literature search.

was significant heterogeneity among the included studies $\left(\mathrm{I}^{2}=0.0 \%, \mathrm{P}=0.962\right)$, so the fixed-effects model was used to combine analysis. The analysis showed that the XST combined with WM group's total effective rate was higher than that of the WM alone group, and there were significant differences between the 2 groups [odds ratio (OR) $=4.04,95 \%$ CI: 2.86 to $5.73, \mathrm{P}<0.001]$ (Figure 3).

\section{NIHSS score}

A total of 9 articles $(20,21,24-28,30,31)$ reported the NIHSS score of ischemic stroke patients after treatment with XST combined with WM, and pooled analysis was performed as a continuous variable. Heterogeneous test analysis showed that there was significant heterogeneity among the included articles $\left(\mathrm{I}^{2}=94 \%, \mathrm{P}<0.001\right)$, so the random-effects model was selected to combine analysis. The results showed that XST combined with WM was more effective in reducing the NIHSS score compared with the WM alone group, and the difference between groups was statistically significant (WMD $=-3.17,95 \%$ CI: -4.14 to $-2.20, \mathrm{P}<0.001$ ) (Figure 4).

\section{Fibrinogen level}

A total of 3 papers $(21,22,27)$ reported the fibrinogen levels of ischemic stroke patients treated with XST. The results of continuous variable comparison analysis showed that there was heterogeneity among the included papers $\left(\mathrm{I}^{2}\right.$ $=88.9 \%>50 \%, \mathrm{P}<0.001)$, so the random-effects model to combine analysis was utilized. The meta-analysis results showed that the fibrinogen level of patients treated with XST combined with WM was significantly lower than that of patients in the WM group (WMD $=-0.73$, 95\% CI: -1.23 to $-0.24, \mathrm{P}<0.001$ ) (Table 2).

\section{Plasma viscosity}

Plasma viscosity was reported in 4 papers $(22,23,25,26)$ and the heterogeneous test analysis showed that there was high statistical heterogeneity $\left(\mathrm{I}^{2}=99.2 \%>50 \%, \mathrm{P}<0.001\right)$, so the data were analyzed using the random-effects model. The meta-analysis results showed that XST combined with WM was more effective in reducing the content of plasma 
Table 1 Characteristics of included trials

\begin{tabular}{|c|c|c|c|c|c|}
\hline Study ID & Age & Sample size (T/C) & Intervention & Outcomes & Duration (d) \\
\hline Wu et al. 2016, (21) & $\mathrm{T}: 58.64 \pm 7.13$ & & $\mathrm{~T}: \mathrm{XST}+$ aspirin & & \\
\hline \multirow[t]{2}{*}{ Xiao et al. 2019, (23) } & C: $58.7 \pm 11.7$ & $65 / 65$ & C: aspirin & (4)(5)(6) & 14 \\
\hline & T: $55.16 \pm 10.92$ & & $\mathrm{~T}: \mathrm{XST}+$ Atorvastatin calcium & & \\
\hline Xu et al. 2018, (24) & C: $55.37 \pm 10.49$ & $51 / 51$ & C: Atorvastatin calcium & (1)(2)(7) & 14 \\
\hline Wang et al. 2017, (25) & $\mathrm{T}: 59.54 \pm 7.33$ & & $\mathrm{~T}: \mathrm{XST}+$ Atorvastatin calcium & & \\
\hline \multirow[t]{2}{*}{ Li et al. 2015, (26) } & C: $58.23 \pm 7.45$ & $62 / 62$ & C: Atorvastatin calcium & (1)(2)(4)(5)(6)(7) & 14 \\
\hline & $\mathrm{T}: 59.2 \pm 7.8$ & & T: XST + Atorvastatin calcium & & \\
\hline \multirow[t]{2}{*}{ Liu et al. 2019, (27) } & C: $59.8 \pm 7.4$ & $48 / 48$ & C: Atorvastatin calcium & (1)(2)(3)(7) & 14 \\
\hline & T: $68.32 \pm 5.63$ & & $\begin{array}{l}\text { T: Sanqi Panax Notoginseng for } \\
\text { Injection + Edaravone Injection }\end{array}$ & & \\
\hline \multirow[t]{2}{*}{ Hu et al. 2017, (28) } & C: $68.59 \pm 5.47$ & $56 / 52$ & C: Edaravone Injection & (1)(2)(5)(6) & 30 \\
\hline & T: $68.28 \pm 7.26$ & & T: XST + Edaravone Injection & & \\
\hline \multirow{2}{*}{ Lin et al. 2017, (31) } & $\mathrm{T}: 62.6 \pm 7.2$ & & $\begin{array}{l}\text { T: XST + Butylphthalide soft } \\
\text { capsules }\end{array}$ & & \\
\hline & C: $61.4 \pm 6.9$ & & C: Butylphthalide soft capsules & & \\
\hline
\end{tabular}

XST, Xuesaitong Injection; T, Treatment group; C, Control group; NIHSS, National Institutes of Health Stroke Scale. Note of outcomes: (1) total effective rate; (2) NIHSS score improvement; (3) fibrinogen; (4) plasma viscosity; (5) high shear viscosity of whole blood; (6) low shear viscosity of whole blood; (7) adverse reactions.

viscosity, and the statistical difference between 2 groups was significant (WMD $=-4.88,95 \% \mathrm{CI}:-8.41$ to -1.35 , $\mathrm{P}=0.007$ ) (Table 2).

\section{Whole blood high shear viscosity}

Whole blood high shear viscosity was reported in 5 papers
$(22,23,25,26,28)$ and the heterogeneous test analysis showed that there was high statistical heterogeneity $\left(\mathrm{I}^{2}=97.0 \%>50 \%\right.$, $\mathrm{P}<0.001$ ), so the random-effects model was selected for combined analysis. The results showed that compared with the control group, the whole blood high shear viscosity of patients in the XST combined with WM group was obviously decreased compared to the WM alone group 


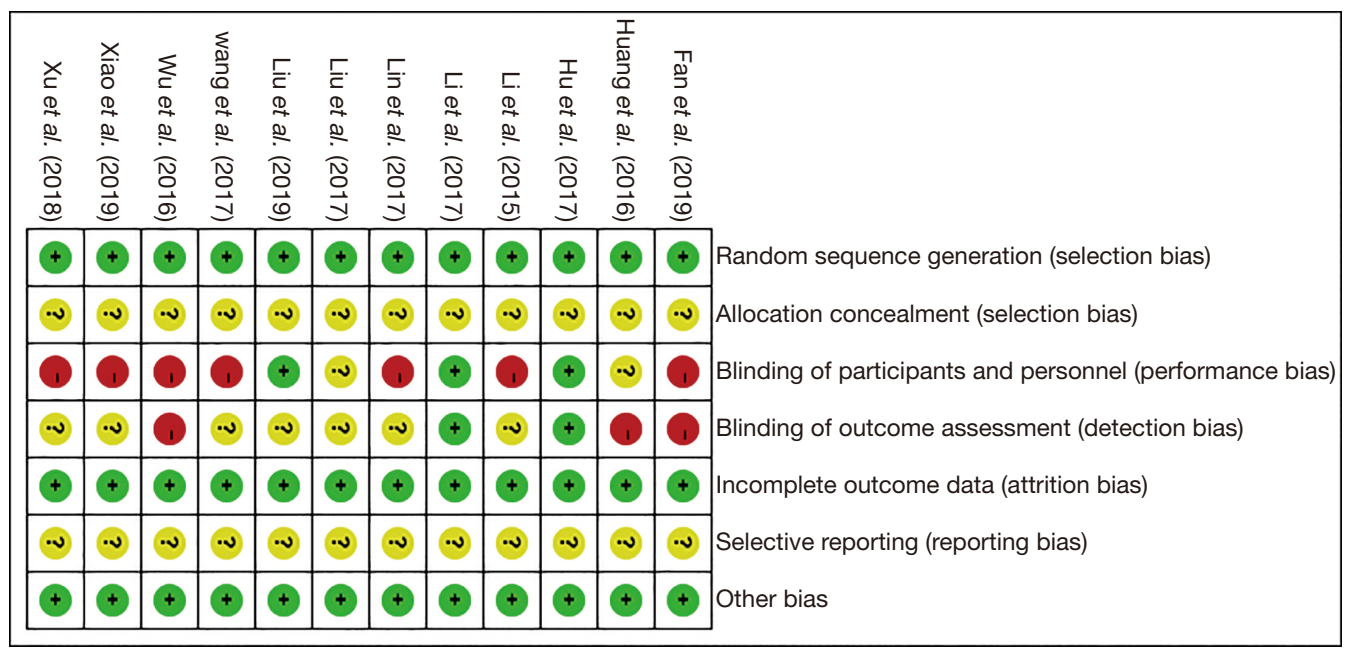

Figure 2 Risk of bias of the included studies. Green, yellow and red color represents low risk, unknown risk and high risk, respectively.

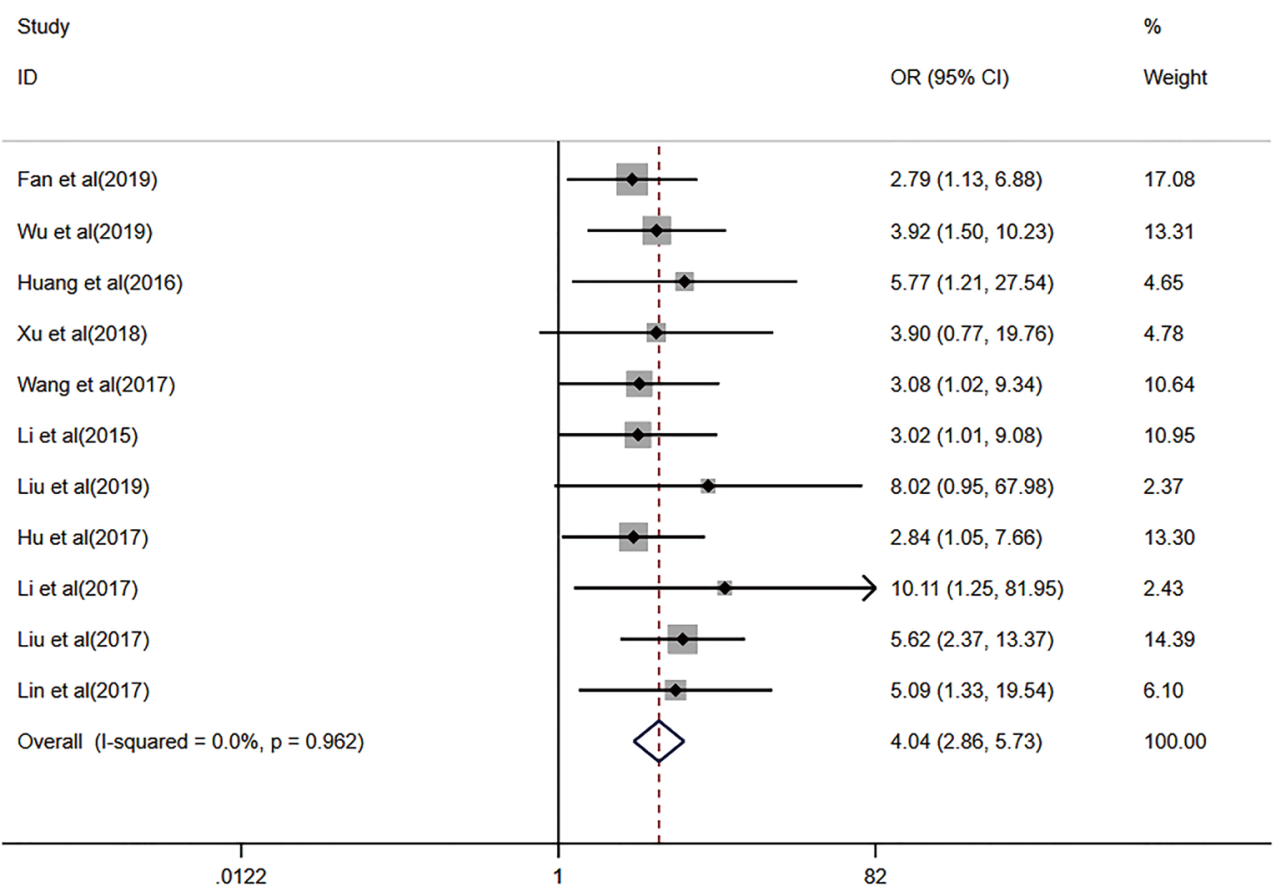

Figure 3 Forest chart of meta-analysis on the total effective rate of 2 groups of patients.

$(\mathrm{WMD}=-1.68,95 \% \mathrm{CI}:-2.78$ to $-0.58, \mathrm{P}=0.003)$ (Table 2).

\section{Whole blood low shear viscosity}

The whole blood low shear viscosity of patients was reported in $5(22,23,25,26,28)$ studies, and the heterogeneity test analysis showed that there was low heterogeneity among the included studies $\left(\mathrm{I}^{2}=43.6 \%<50 \%, \mathrm{P}=0.131\right)$, so the data were analyzed using the fixed-effects model. The results showed that the whole blood low shear viscosity of patients in the XST combined with WM group was significantly lower than that in the WM alone group (WMD 


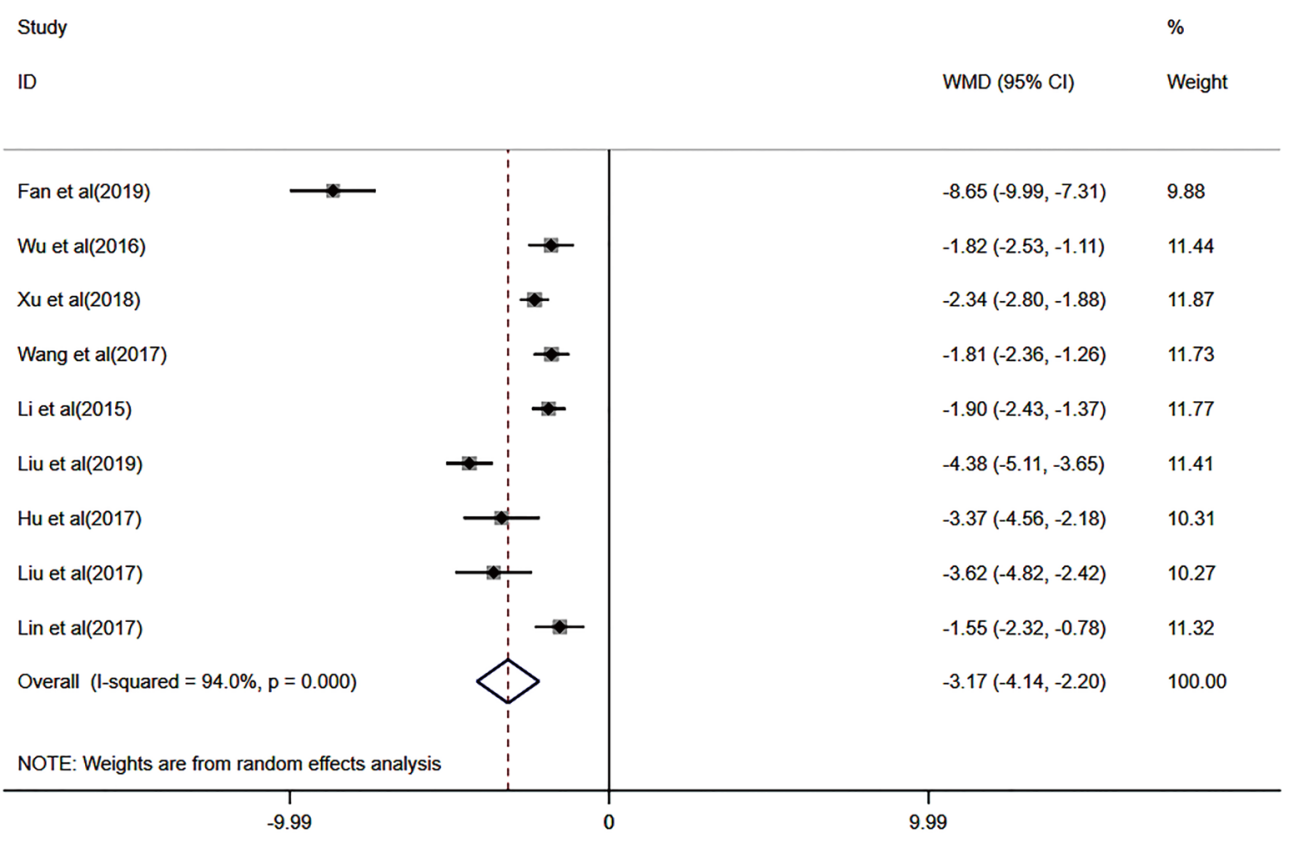

Figure 4 Meta-analysis of NIHSS Score Improvement in Two Groups of Patients. NIHSS, National Institutes of Health Stroke Scale.

Table 2 Meta-analysis results of fibrinogen level, plasma viscosity, whole blood high shear viscosity, whole blood low shear viscosity in 2 groups of ischemic stroke patients

\begin{tabular}{|c|c|c|c|c|c|c|c|}
\hline \multirow{2}{*}{ Outcomes } & \multirow{2}{*}{ Studies (n) } & \multirow{2}{*}{ Cases (n) } & \multicolumn{2}{|c|}{ Heterogeneity test } & \multirow{2}{*}{ Effect model } & \multirow{2}{*}{ WMD (95\% Cl) } & \multirow{2}{*}{$P$ value } \\
\hline & & & $I^{2}$ & $P$ value & & & \\
\hline Fibrinogen level & 3 & 420 & $88.9 \%$ & $<0.001$ & Random & $-0.73(-1.23,-0.24)$ & 0.004 \\
\hline Plasma viscosity & 4 & 490 & $99.20 \%$ & $<0.001$ & Random & $-4.88(-8.41,-1.35)$ & 0.007 \\
\hline $\begin{array}{l}\text { Whole blood high shear } \\
\text { viscosity }\end{array}$ & 5 & 598 & $97.00 \%$ & $<0.001$ & Random & $-1.68(-2.78,-0.58)$ & 0.003 \\
\hline
\end{tabular}

WMD, weighted mean difference; $\mathrm{Cl}$, confidence interval.

$=-0.81,95 \%$ CI: -0.97 to $-0.64, \mathrm{P}<0.001)$ (Table 2).

\section{Adverse reactions}

Among the 12 included articles, a total of $8(15,17,18,21,24$, $26,27,31)$ reported the occurrence of adverse reactions of XST combined with WM. There were 2 from 5 studies that reported pruritus, 2 reported gastrointestinal discomfort, 1 study reported dizziness and headache, 3 studies reported other adverse reactions. Meta-analysis results showed that the incidence of adverse reactions in patients in the XST combined with WM group was not significantly different from that in the WM alone group, as shown in Table 3.

\section{Sensitivity analysis}

Sensitivity analysis of the total effective rate was carried out by excluding any single study each time to test the study effect on pooled effect size, and we found that there was no qualitative change in the combined effect. Therefore, the pooled results of included study were robust, as shown in Figure 5. 
Table 3 Meta-analysis results of adverse reactions in 2 groups of patients

\begin{tabular}{|c|c|c|c|c|c|}
\hline Adverse reaction & Studies (n) & \multicolumn{2}{|c|}{ Participants (n) } & OR (95\% Cl), $\mathrm{P}$ value & References \\
\hline Gastrointestinal discomfort & 2 & 96 & 96 & 0.27 (0.06-1.15), 0.076 & $(24,31)$ \\
\hline Dizziness, headache & 1 & 48 & 48 & 2.04 (0.18-23.32), 0.565 & (27) \\
\hline Pruritus & 2 & 145 & 145 & 1.61 (0.20-13.29), 0.656 & $(21,31)$ \\
\hline
\end{tabular}

T, Treatment group; C, Control group; OR, odds ratio; Cl, confidence interval.

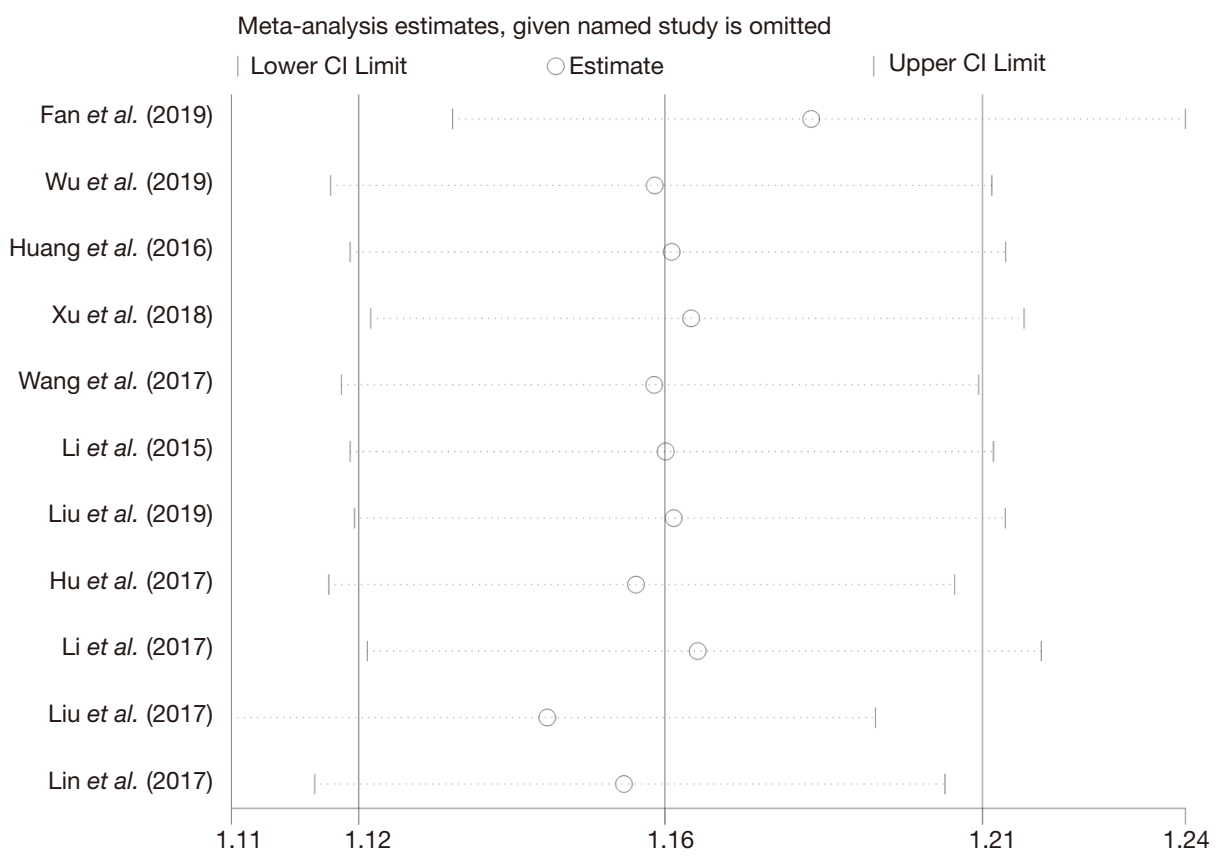

Figure 5 Sensitivity analysis of the total effective rate.

\section{Publication bias}

The funnel plot showed that there was no publication bias in the included studies, and Begg's test $(\mathrm{P}=0.087)$ and Egger's test $(\mathrm{P}=0.08)$ indicated that there was no obvious publication bias. Publication bias results of the included studies were shown in Figure 6.

\section{Conclusions}

Pooled analysis of current clinical evidences showed that the combination of XST and WM has a better curative effect in ischemic stroke and can significantly improve hemorheology (reduce fibrinogen level and whole blood viscosity) compared WM alone. Meanwhile, our study found that the occurrence of adverse reactions in the XST group was not significantly different to that of the control group, which was consistent with the results of previous meta-analyses $(12,13)$. Compared with the previous meta-analysis, our study had the following advantages: (I) the inclusion of more high-quality and recently published studies; (II) sample size was more than 45 cases; (III) the control group was treated with WM to reduce the heterogeneity of metaanalysis. Therefore, this meta-analysis is more scientific and accurate than any previous such studies.

In our study, the results of pooled-analysis showed that the degree of reduction of NIHSS score in the XST combined with WM group was significantly better than that in the WM alone group (WMD =-3.35, 95\% CI: 


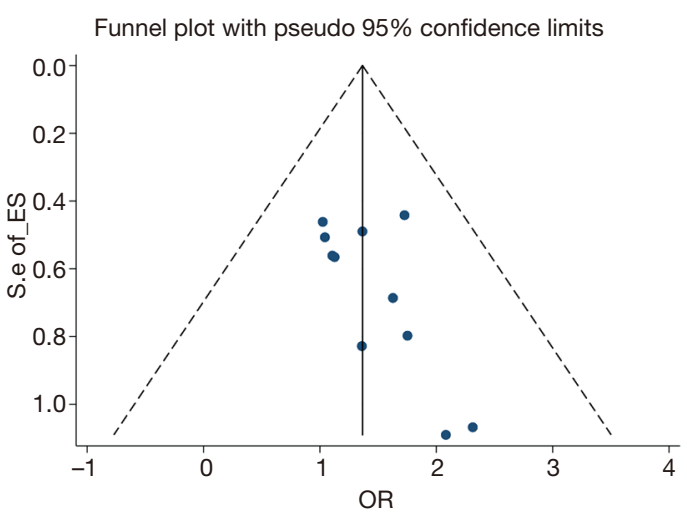

Figure 6 Funnel plot of included studies.

-4.30 to $-2.40, \mathrm{P}=0.000$ ), that is, NIHSS score in mild stroke patients recovered to a normal level, and severe stroke patients recovered to mild or moderate stroke level after the treatment of XST combined with WM. The study by Qiao (16), also included in this meta-analysis, found that after XST combined with Aspirin treatment, the NIHSS score of patients with senile ischemic stroke changed from $25.76 \pm 8.92$ to $9.55 \pm 4.68$, and the NIHSS score changed from $25.91 \pm 8.86$ to $18.20 \pm 6.97$ in aspirin treatment group. A study by Li et al. (15), showed that the NIHSS score decreased from $15.68 \pm 3.84$ to $5.45 \pm 1.31$ in cerebral infarction patients treated with XST combined with edaravone; however, in the edaravone treatment group, the NIHSS score decreased from $15.73 \pm 3.75$ to $9.83 \pm 2.21$. Therefore, XST combined with WM treatment has a better therapeutic effect compared to treatment with WM alone.

When ischemic stroke occurs, a large amount of oxygenfree radicals and malonaldehyde (MAD) is generated, and directly damages nerve cells; simultaneously, excessive glutamic acid release and blocked reuptake cause cascade reaction, leading to neurotoxic injury. At the same time, cerebral ischemia seriously affects the oxygen supply function of mitochondria and accelerates cell damage. The XST agent protects nerve cells against damage caused by ischemia through expanding blood vessels to increase cerebral blood supply, reduce intracranial pressure, increase SOD level to scavenge oxygen free radicals, and reduce MAD level in brain tissue. Therefore, XST agent can effectively improve the patients' neurological deficit and their quality of life. In a study of 65 patients with acute cerebral infarction, Xiao et al. (23) found that the treatment of XST significantly improved cerebral blood flow, blood viscosity, and hemorheology, and had superior outcomes to the atorvastatin calcium combined atorvastatin calcium treatment $(\mathrm{P}<0.05)$. In this study, we found that XST combined with WM could improve fibrinogen level and blood viscosity in patients. Therefore, the TCM agent, XST, combined with WM, can effectively improve hemorheology, and inhibit thrombosis for patients with ischemic stroke.

Obvious changes in hemorheology are important indicators for evaluating the severity of ischemic stroke. Due to visible components such as fibrinogen in blood, thrombosis is formed at the damaged intima of blood vessels by promoting the aggregation of platelets and red blood cells, reducing blood flow velocity, and causing blood to be in a state of hypercoagulability and high viscosity $(32,33)$. Besides, the formation of necrotic cells, reactive oxygen species (ROS), and activated inflammatory cells can initiate inflammatory reactions and finally affect the prognosis of ischemic stroke.

The XST agent can promote the dissolution of fibrinogen, inhibit platelet aggregation due to elevated adenosine diphosphate (ADP) level, reduce whole blood viscosity, improve microcirculation, and inhibit thrombosis (34). The main components of XST, Panax notoginsenosides, and so on (35), could reduce blood viscosity by dilating peripheral blood vessels and regulating blood lipid, improve the high shear and low shear viscosity levels of whole blood, and at the same time XST agent could reduce inflammatory reaction to a certain extent, protect vascular endothelial cells, and inhibit thrombosis. This mechanism has been verified in pharmacological experiments and several clinical studies $(36,37)$.

In the pharmacological experiments, XST agent can effectively inhibit platelet aggregation and thrombosis formation (38), effects which may arise from the effective antiplatelet aggregation effects of the main components of the XST agent, including notoginsenoside R1, ginsenoside $\mathrm{Rg} 1, \mathrm{Rb} 1, \mathrm{RD}$, and RE $(5,6,39)$. In a clinical study by Sun et al. (40), XST was shown to significantly decrease mean platelet volume (MPV) after 2 weeks, compared with conventional treatment $(\mathrm{P}<0.05)$. In a clinical study by Huang et al. (22), after treatment with XST, the prothrombin time, fibrinogen level, and D-dimer level of acute cerebral infarction patients were significantly improved, which was significantly better than that of the control group (aspirin only) $(\mathrm{P}<0.05)$. Therefore, XST agent can effectively inhibit platelet aggregation and thrombosis.

The results of this study showed that XST combined with 
WM can effectively reduce fibrinogen level, prevent platelet aggregation, improve microcirculation, inhibit thrombosis, significantly improve the neurologic impairment of patients, and improve the quality of life of ischemic stroke patients. Therefore, combined use of XST with WM agent can better improve the cerebral microcirculation of patients and effectively inhibit thrombosis, providing a choice for clinical medication. The main adverse reactions of XST are gastrointestinal discomfort, dizziness, headache, pruritus, and rash. This meta-analysis showed that the incidence of adverse reactions in the XST group (17 cases, and 169 cases) was similar to the incidence rate of adverse reactions in the WM alone group (19 cases, and 169 cases, 3.6\% vs. 4.1\%), and gastrointestinal discomfort was the most frequently experienced adverse reaction (accounting for $48.3 \%$ of the total adverse reactions). These adverse reactions may have been caused by the binding of TCM components to conjugated protein in vivo $(5,6,39)$, and may also be related to the dosage of XST. Overall, the use of XST has higher safety in clinical application, and patients treated with XST still need to be carefully monitored for adverse reactions.

\section{Limitation}

This meta-analysis still had some limitations: (I) considering that the value of meta-analysis depends on the quality of the included studies and the methodological quality of bias control, the limitation of our study was that most of the literature on TCM was from China, while literature from other countries was few, which may have led to publication bias. (II) Some researchers may have selectively rejected negative results or some adverse results, resulting in publication bias. (III) At present, there are little research data on the anti-platelet aggregation of XST. (IV) The measures of patients' neurological deficit degree were inconsistent, with NIHSS score used in some studies and Barthel index (BI) or other scores used in other studies. (V) Clinical heterogeneity (caused by different control groups receiving different western medicine treatments) can be assessed by subgroup analysis. Therefore, it is suggested that more rigorous clinical studies should be carried out in the future, such as large-scale randomized double-blind trials. Endpoints should be strictly standardized, such as evaluating the platelet data, and establishing a unified score for the evaluation of patients' physical condition. Standardized training could be provided to researchers to better evaluate the therapeutic effect of XST.

In summary, comprehensive analysis of articles showed that XST combined with WMs is an effective treatment strategy for the treatment of ischemic stroke, which improved the total clinical effective rate, promoted the recovery of patients' neurological function, and had few adverse reactions and high safety in clinical application. Therefore, this meta-analysis has a guiding significance for clinical application in acute ischemic stroke therapy.

\section{Acknowledgments}

Funding: This research was supported by the Science and Technology Department of Sichuan Province (2020YFS0155). The funding body played an important role in the data retrieval and revision of the article.

\section{Footnote}

Reporting Checklist: The authors have completed the PRISMA reporting checklist. Available at https://dx.doi. org/10.21037/apm-21-1828

Conflicts of Interest: All authors have completed the ICMJE uniform disclosure form (available at https://dx.doi. org/10.21037/apm-21-1828). The authors have no conflicts of interest to declare.

Ethical Statement: The authors are accountable for all aspects of the work in ensuring that questions related to the accuracy or integrity of any part of the work are appropriately investigated and resolved.

Open Access Statement: This is an Open Access article distributed in accordance with the Creative Commons Attribution-NonCommercial-NoDerivs 4.0 International License (CC BY-NC-ND 4.0), which permits the noncommercial replication and distribution of the article with the strict proviso that no changes or edits are made and the original work is properly cited (including links to both the formal publication through the relevant DOI and the license). See: https://creativecommons.org/licenses/by-nc-nd/4.0/.

\section{References}

1. Wu Z, Li ZR, Dai YQ, et al. Relationship between risk perception and lifestyle in ischemic stroke patients with H-type hypertension. Ann Palliat Med 2020;9:3731-41.

2. Shen SY, Fu XD, Qian SF, et al. The explanation and development of intervention strategy of ischemic brain 
protection drugs in combination of traditional Chinese and Western Medicine. Chin J Infor on Trad Chin Med 2005;12:7-8.

3. Gao Y, Zhou L. Research progress and present situation on wind stroke for the prevention and treatment of TCM. Glo Trad Chin Med 2009;2:15-8.

4. Peng X, Luo C. Research progress of traditional Chinese and western medicine in the treatment of hemorrhagic stroke by promoting blood circulation and removing blood stasis. J Emer in Trad Chin Med 2017;26:845-7.

5. Allard CB, Scarpelini S, Rhind SG, et al. Abnormal coagulation tests are associated with progression of traumatic intracranial hemorrhage. J Trauma 2009;67:959-67.

6. Retraction note to: Ginsenoside-Rg1 enhances angiogenesis and ameliorates ventricular remodeling in a rat model of myocardial infarction. J Mol Med (Berl) 2013;91:645.

7. Sun F. Efficacy of TCM on neurological deficits and disability rate in patients with acute ischemic stroke. Clin J of Chin Med 2019;11:67-9.

8. Ma WN, Yu P, Yang YF, et al. Research progress of the effect of Xuesaitong and its chemical components on CYP450 enzyme. World J Int Trad and West Med 2018;13:1030-3,6.

9. Weng YL, Huang LI, Ting WU, et al. Overview of the Research on the Treatment of Cerebral Apoplexy with the Effective Ingredients of Traditional Chinese Medicine. Strait Phar J 2019;31:33-7.

10. Xu J, Wang J, Wen LJ. Overview of the study on the intervention of total saponins of notoginseng on thrombosis. Yunnan J Trad Chin Med and Mate Med 2003;24:46-7.

11. Han SX, You Y. Balance between cardiovascular pharmacological and hemolytic effects of saponins of Panax notogenseng. Zhongguo Zhong Yao Za Zhi 2016;41:818-22.

12. Zhang XM, Wu JR, Zhang B. Xuesaitong injection as one adjuvant treatment of acute cerebral infarction: a systematic review and meta-analysis. BMC Complement Altern Med 2015;15:36.

13. Duan XJ, Zhang D, Wang KH, et al. Comparative study of xuesaitong injection and compound salvia miltiorrhizae injection in the treatment of acute cerebral infarction: a meta-analysis. J Int Med Res 2019;47:5375-88.

14. Liu N, Chen Y, Zhuang F, et al. Meta-analysis of the Efficacy and Safety of Xuesaitong Injection Combined with Ozagrel Injection on Acute Cerebral Infarction. J
Emer in Trad Chin Med 2019;28:382-6.

15. Li N, Meng F, Gao F, et al. A Randomized Controlled Trial of Xuesaitong for Injection in the Treatment of Stagnation of Blood Stasis Syndrome during Cerebral Infarction. World Chin Med 2019;14:395-8.

16. Qiao HQ. Effect of Xuesaitong Injection on nerve function, coagulation and fibrinolysis system in patients with acute cerebral infarction. J Huaihai Med 2018;36:683-5.

17. Wang DH, Bin MA, Sun CY, et al. Multi-center clinical study of Xuesaitong Injection (lyophilization) in treatment of cerebral infarction recovery period (blood stasis syndrome). Drugs \& Clinic 2017;32:1864-71.

18. Li CS, Kong L, Yuan FL, et al. The effect of Xuesaitong Injection on the elderly with ischemic stroke and the quality of life. Chinese J Gero 2016;36:4183-4.

19. Li RL, Ye ZM, Ling ZA. Effect of Xuesaitong Injection on cellular immune function and high sensitive C-reactive protein in patients with acute cerebral infarction. J Guangxi Med University 2015;32:758-60.

20. Fan RR, Zhao JH, Ma MM, et al. Effect of Xuesaitong injection combined with aspirin on senile ischemic stroke and serum Thl/Th2 cytokine level. Shaanxi J Trad Chin Med 2019;40:1032-5.

21. Wu YJ. Effect of aspirin combined with Xuesaitong injection on the serum Hcy levels of 100 cases of patients with ischemic stroke. Shanghai Med \& Phar J 2016;37:44-7.

22. Huang Y. Effect of Xuesaitong Injection on the curative effect and thrombosis of acute cerebral infarction. Chin J Prac Ner Dis 2016;19:102-3.

23. Xiao J, Feng JL, Rong TY, et al. Effect of Xuesaitong Injection on electrocardiographic changes and hemorheology in patients with acute cerebral infarction. Modern J Inte Trad Chin and Western Med 2019;28:202-5.

24. $\mathrm{Xu} \mathrm{W}$. Observation on the therapeutic effect of Xuesaitong injection combined with atorvastatin calcium on diabetes mellitus with cerebral infarction. Healthy People 2018;12:129-30.

25. Wang BJ, Han XF, Guo AH. Effect of atorvastatin calcium combined with Xuesaitong Injection on Hemorheology and nerve function in patients with diabetes mellitus and cerebral infarction. Shaanxi Med J 2017;46:533-4.

26. Li MC, Liao CQ, Yang XP. Efficacy of Xuesaitong injection combined with Atorvastatin Calcium in treating diabetes mellitus complicated with cerebral infarction and its effects on neurological function. China J Mod Med 
2015;25:49-52.

27. Liu BG, Zhang R, Xie N, et al. Curative effect of Xuesaitong combined with edaravone on patients with cerebral infarction. Hainan Med J (Chin) 2019;30:2625-8.

28. Hu YZ, Pharmacy DO, Hospital PP. Clinical curative effect of Xuesaitong Injection combined with edaravone in treatment of elderly patients with acute cerebral infarction and influence on Hemorheology of patients with plasma C reactive protein. J Qiqihar University of Med 2017;38:170-2.

29. Li DH. Clinical effect of Xuesaitong combined with edaravone in the treatment of cerebral infarction. China J Phar Econ 2017;12:61-3.

30. Liu LL. Observation on the effect of the combination of Xuesaitong and edaravone injection on 120 patients with cerebral infarction. Guide of China Med 2017;15:199-200.

31. Lin LH, Chen Y. Effect of Xuesaitong injection in adjuvant treatment of acute cerebral infarction and its impact on neurological function. Chin J Mod Drug App 2017;11:17-9.

32. de Moerloose P, Casini A, Neerman-Arbez M. Congenital fibrinogen disorders: an update. Sem Thro Hem 2013;39:585-95

33. Zhang HJ, Shao CX, Guo WL. Relationship between high sensitive C-reactive protein, fibrinogen and the stability

Cite this article as: Feng $\mathrm{L}, \mathrm{Wu} \mathrm{XJ}$, Cao T, $\mathrm{Wu}$ B. The efficacy and safety of Xuesaitong injection combined with western medicines in the treatment of ischemic stroke: an updated systematic review and meta-analysis. Ann Palliat Med 2021;10(9):9523-9534. doi: 10.21037/apm-21-1828 of carotid atherosclerotic plaque in patients with ischemic stroke. Chin J Coal Industry Med 2014;17:89-91.

34. Lin Y, Qiu KF, Wu JY. Meta analysis of therapeutic effect of Xuesaitong Injection on cerebral infarction. China J Phar Econ 2012:176-8.

35. Wang G, Chen Q, Li ZF, et al. Study Progress on Mechanism of Protective Effect of PNS and Its Major Components on Brain Cell. Chin Arch Trad Chin Med 2017;35:1743-6.

36. Feng XY, Chen WH, Wu XY, et al. Study on the effect of Panax Notoginsenoside on lipid-lowering and no in NAFLD rats. Mod Trad Chin Med 2018;38:140-3.

37. Zhang JF, Zhang DS. Study Advancement in Pharmalcological Actions of Total saponins of Panax Notoginseseng. Hunan J Trad Chin Med 2017;33:216-8.

38. Wang LL, Yu YR, Yang JH, et al. Dissecting Xuesaitong's mechanisms on preventing stroke based on the microarray and connectivity map. Mol Biosyst 2015;11:3033-9.

39. Chai H, Dong YL, Wang XW, et al. Ginsenoside $\mathrm{Rb} 1$ attenuates homocysteine-augmented guidewire injury-induced intimal hyperplasia in mice. J Surg Res 2009;157:193-8.

40. Sun QS. Effect of Xuesaitong acupuncture on platelet parameters and hemorheology in 336 patients with ischemic cerebral infarction. J North Phar 2019;16:71-2. 